\title{
Dynamic nomogram for long-term survival in patients with non-small cell lung cancer after pneumonectomy
}

\author{
Zi-Ming Wang ${ }^{1 \wedge}$, Marc Swierzy ${ }^{1}$, Dany Balke ${ }^{1}$, Dania Nachira ${ }^{2}$, Diego González-Rivas ${ }^{1,3,4}$, \\ Harun Badakhshi ${ }^{5}$, Mahmoud Ismail ${ }^{1 \wedge}$
}

${ }^{1}$ Department of Thoracic Surgery, Klinikum Ernst von Bergmann Potsdam, Academic Hospital of the Charité-Universitätsmedizin Humboldt University Berlin, Potsdam, Germany; ${ }^{2}$ Department of General Thoracic Surgery, Fondazione Policlinico Universitario “A. Gemelli”, Rome, Italy; ${ }^{3}$ Department of Thoracic Surgery, Shanghai Pulmonary Hospital, Tongji University School of Medicine, Shanghai, China; ${ }^{4}$ Department of Thoracic Surgery and Minimally Invasive Thoracic Surgery Unit, Coruña University Hospital, Coruña, Spain; ${ }^{5}$ Department of Radiation Oncology, Klinikum Ernst von Bergmann Potsdam, Academic Hospital of the Charité-Universitätsmedizin Humboldt University Berlin, Potsdam, Germany

Contributions: (I) Conception and design: ZM Wang, M Ismail; (II) Administrative support: D González-Rivas, H Badakhshi, M Ismail; (III) Provision of study materials or patients: ZM Wang, D Nachira; (IV) Collection and assembly of data: ZM Wang, M Swierzy; (V) Data analysis and interpretation: ZM Wang, D Balke; (VI) Manuscript writing: All authors; (VII) Final approval of manuscript: All authors.

Correspondence to: Mahmoud Ismail, MD. Director, Department of Thoracic Surgery, Klinikum Ernst von Bergmann, Charlottenstraße 72,14467 Potsdam, Brandenburg, Germany, Email: mahmismail@gmail.com.

Background: The study aims to identify prognostic factors of overall survival (OS) in patients who had pneumonectomy, in order to develop a practical dynamic nomogram model.

Methods: A total of 2,255 patients with non-small cell lung cancer (NSCLC) who underwent pneumonectomy were identified from 2010-2015 in the Surveillance, Epidemiology, and End Results (SEER) database. The cohort was divided into a training (2011-2015) and a validation [2010] cohort. A nomogram and a risk classification system were constructed from the independent survival factors in multivariable analysis. The predictive accuracy of the nomogram was measured through internal and external validation.

Results: Independent prognostic factors associated with OS were gender, age, pathology, tumor size, $\mathrm{N}$ stage, chemotherapy, and radiotherapy. The C-index of the nomogram for OS was 0.675 (95\% CI: 0.655-0.694). Similarly, the AUC of the model was 0.733, 0.709, and 0.701 for the 1-, 3-, and 5-year OS, respectively. The calibration curves for survival demonstrated good agreement. Significant statistical differences were found in the OS of patients within different risk groups. An online calculation tool was established for clinical use.

Conclusions: This novel nomogram was able to provide a reliable prognosis for survival in patients with NSCLC undergoing pneumonectomy.

Keywords: Nomogram; pneumonectomy; non-small cell lung cancer (NSCLC); prognosis

Submitted Nov 01, 2020. Accepted for publication Mar 05, 2021.

doi: $10.21037 /$ jtd-20-3203

View this article at: http://dx.doi.org/10.21037/jtd-20-3203

\section{Introduction}

Pneumonectomy was first successfully performed by Dr. Evarts Graham in 1933 to treat lung cancer, and it has been used as a radical surgical treatment ever since (1). Advances in medical technology and instruments have permitted wide acceptance of lobectomy, segmentectomy, and other surgical methods. Recently, sleeve lobectomy and pulmonary arterioplasty have also demonstrated similar oncological

^ ORCID: Zi-Ming Wang, 0000-0002-7882-0047; Mahmoud Ismail, 0000-0002-4117-0204. 
benefits as pneumonectomy in selected anatomically feasible patients (2). Pneumonectomy is performed in approximately $8.3 \%$ to $15 \%$ of all lung cancer surgeries $(3,4)$. The procedure results in comparatively lower postoperative quality of life, and higher rates of complication, morbidity, and mortality than other surgical approaches. However, this operation is still the radical treatment strategy in centrally located or hilum invaded non-small cell lung cancer (NSCLC) $(5,6)$.

The indications for pneumonectomy should be carefully considered because the procedure requires an extensive removal of lung tissue. Accordingly, accurate assessment of risk factors and the prediction of postoperative survival are of great importance for patients who are candidates for pneumonectomy. Due to the low proportion of pneumonectomy in thoracic surgery and the high postoperative risk, any prospective randomized controlled study can be challenging (7). However, retrospective analysis of the national database on prognostic factors may shed some light. This aggressive surgical approach makes it rare to study the prognostic factors on a large scale or to construct prospective studies $(8,9)$. In recent years, nomograms are widely used for predicting prognosis. These predictive models generate individual quantified probabilities of clinical events by integrating prognostic clinicopathological variables. They are useful statistical prognostic models that bring us closer to achieving personalized medicine. Multiple studies have reported that nomogram scoring systems could provide an accurate prognosis of the disease (10-12).

In this study, we used the Surveillance, Epidemiology, and End Results (SEER) Program to analyze the prognostic factors of lung cancer patients who underwent pneumonectomy. A predictive dynamic nomogram was constructed to evaluate the survival and prognosis of patients with different clinical and pathological characteristics in the training set. The evaluation and verification of the model were carried out with data of the training and verification set, respectively.

We present the following article in accordance with the TRIPOD reporting checklist (available at http://dx.doi. org/10.21037/jtd-20-3203).

\section{Methods}

\section{Study population}

Data in the study were acquired from the SEER database of the National Cancer Institute in the United States. The registry data program collects and publishes cancer incidence and survival data from population-based cancer registries covering approximately $34.6 \%$ of the United States population (https://seer.cancer.gov/data/). The SEER*Stat software (version 8.3.8) was used to access the Incidence - SEER 18 Regs Custom Data (with additional treatment fields), Nov 2018 Sub (1975-2016 varying). Patients in the database were included if they had: (I) histology codes (International Classification of Diseases for Oncology, third edition, ICD-O-3) for adenocarcinoma: $8140,8144,8230,8244,8250-8255,8260,8310,8323$, $8333,8470,8480,8481,8490,8550,8551,8574$; squamous cell carcinoma: 8050, 8052, 8070-8074, 8083, 8084, 8123; neuroendocrine carcinoma: $8013,8240,8246,8249$; or other NSCLC: 8012; 8022; 8030-8033; 8035; 8046; 8051; 8082; 8200; 8430; 8560 (13); (II) "Primary Site-labeled" of any $I C D-O-3$ value ranging from $\mathrm{C} 34.0$ to $\mathrm{C} 34.9$ for a primary tumor site in the lung; (III) "Histology" with positive confirmation by diagnostic pathology; and (IV) "Therapy. Rx Sum--Surg Prim Site (1998+)" with values "55, 56, 65, 66, 70" for pneumonectomy. Furthermore, we selected patients who were diagnosed from 2010 to 2015 using the $7^{\text {th }}$ AJCC Staging System. Patients with a diagnosis only confirmed by autopsy, 30-day operative mortality, or distant metastasis (M1) were excluded. Complete-case analysis is used to handle the missing data. The study was conducted in accordance with the Declaration of Helsinki (as revised in 2013).

\section{Variables}

Baseline demographic variables included age, gender, race, year of diagnosis, marriage, vital status, cause of death, and survival months. Tumor descriptors included laterality, histopathologic subtypes, grading, tumor size, T stage, number of lymph nodes dissection (LND), number of lymph nodes positive (LNP), N stage, TNM stage, surgery type, and additional therapy (e.g., chemotherapy and radiotherapy). According to the SEER variables dictionary, radical pneumonectomy was defined as pneumonectomy with mediastinal lymph node dissection, and extended pneumonectomy was radical pneumonectomy with the dissection of surrounding structures such as the diaphragm, pleura, and chest wall. The primary endpoint was overall survival (OS). The variable "Survival months" was used to identify survival time. OS was calculated by the difference in months from the diagnosis to death due to any reason listed 
under the variable "Vital status recode". This study was a registry population-based research, so it did not involve blinding of research analysis and outcome or adjusted association between each candidate predictor and outcome.

\section{Predictive model}

According to the statement of transparent reporting of a multivariable prediction model for individual prognosis or diagnosis (TRIPOD), the authors set eligible patients diagnosed in 2011-2015 as the internal training cohort, and the patients diagnosed in 2010 as the external validation cohort (14). The training cohort was used to establish the predictive model and to develop the nomogram. The validation cohort was used to validate this model. The Cox proportional hazards model was used for the univariate analysis of the OS in the training cohort. Any univariate result with a $\mathrm{P}$ value less than 0.05 was entered into a multivariate analysis. A hazard ratio (HR) and a corresponding $95 \%$ confidence interval (CI) were calculated. Based on results of the multivariate analysis, a nomogram for OS was constructed from its independent prognostic factors. Harrell's concordance index (C-index) and the area under the time-dependent receiver operating characteristic curve (AUC) were used to measure the performance of the nomogram. Internal calibration plots with 400 bootstrap resamples from the training cohort was used to evaluate the nomogram performance, which compared the predicted and observed probabilities of 1-, 3 -, and 5-year OS. The "rms" package was used to perform the external validation in the test cohort with the same predictors for the nomogram model from the training set. We divided the training group into high-risk group and low-risk group according to the median value of the total score for each patient, and the OS differences between lowand high-risk groups in the validation and total cohort were also evaluated. The authors analyzed the risk classification system based on the nomogram model to distinguish highrisk and low-risk groups in the enrolled patients with stage I-III NSCLC after pneumonectomy. An easy-to-use web nomogram model calculation was developed at https:// shinyapps.io/ and the most updated version will also be available online.

\section{Statistical analyses}

Differences in continuous data were analyzed by the MannWhitney $U$ test or Student's $t$-test. Categorical variables were analyzed by Chi-squared or Fisher's exact tests, as appropriate. Kaplan-Meier graphs were created for survival analysis. Statistical analyses were performed by SPSS (Version 24.0; IBM Corporation, Armonk, NY, USA). R Project (Version 4.0.3) was used to build and evaluate the performance of the nomogram. "Rms" package was used to build and evaluate the nomogram model. "Shiny" package was used to develop the dynamic version. All statistical tests were two-tailed, and a $\mathrm{P}$ value less than 0.05 was considered as statistically significant.

\section{Results}

\section{Patient characteristics}

Pneumonectomy accounts for about 3.9\% $(2,583 / 64,874)$ of all lung malignant tumor resections performed in the SEER database within the same study period. The proportion of pneumonectomy was reduced from $4.7 \%$ to $3.1 \%$ during 2011-2015. A total of 2,255 patients who met the inclusion criteria were included in the study. Among these patients, $62.5 \%$ were male, and the median age was 63 years old (IQR, 56-70 years old). Squamous cell carcinoma was the most common pathological type, accounting for $48.0 \%$. The median tumor size was $5.0 \mathrm{~cm}$ (IQR, $3.2-7.0 \mathrm{~cm}$ ). Lymph node metastasis was found in $59.6 \%$ of patients. Pneumonectomy was performed on the left side in $60.1 \%$ of the patients. Among all the patients, $70.5 \%$ underwent radical pneumonectomy including mediastinal lymph node dissection. Chemotherapy was given in $53.5 \%$ of the patients and $20.6 \%$ received radiotherapy. The training group contained $1,846(81.9 \%)$ patients and the verification group contained 409 (18.1\%) patients. Clinicopathological characteristics of the patients between the training group and the verification group are shown in Table 1 .

\section{Survival analyses and prediction model}

The 1-, 3-, and 5-year OS of pneumonectomy were 75.9\%, $54.9 \%$, and $44.4 \%$, respectively. The estimated median OS time was 46.0 months for the whole cohort. The median follow-up time was 44.5 months. As shown in Table 2, the univariate Cox regression model revealed that gender, age, pathology type, grading, laterality, tumor size, $\mathrm{N}$ stage, number of LNP, chemotherapy, and radiotherapy were significantly associated with OS. Multivariate analysis further confirmed that gender, age, pathology type, laterality, tumor size, $\mathrm{N}$ stage, chemotherapy, and 
Table 1 Patient characteristics in the study $(\mathrm{N}=2,255)$

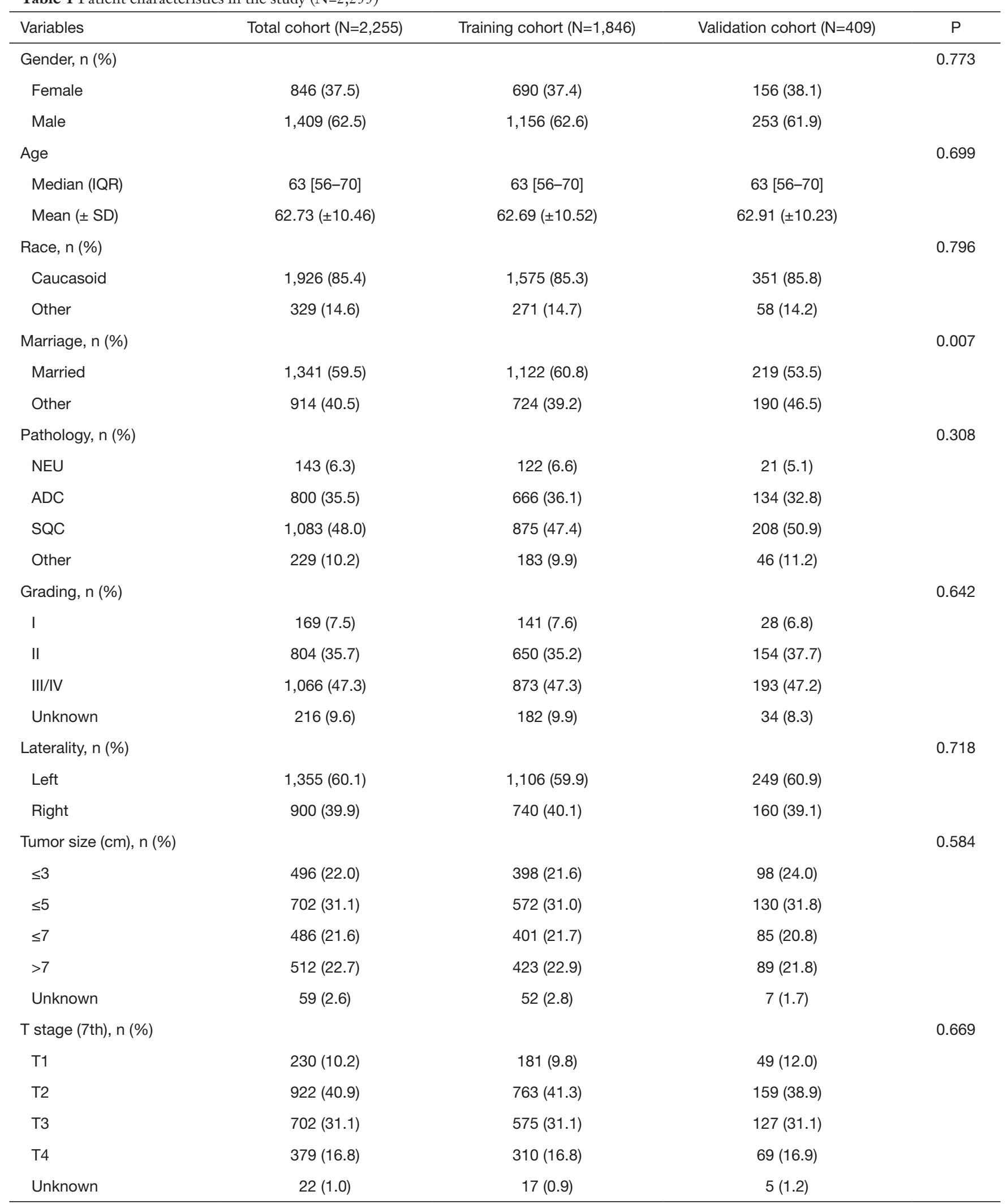

Table 1 (continued) 
Table 1 (continued)

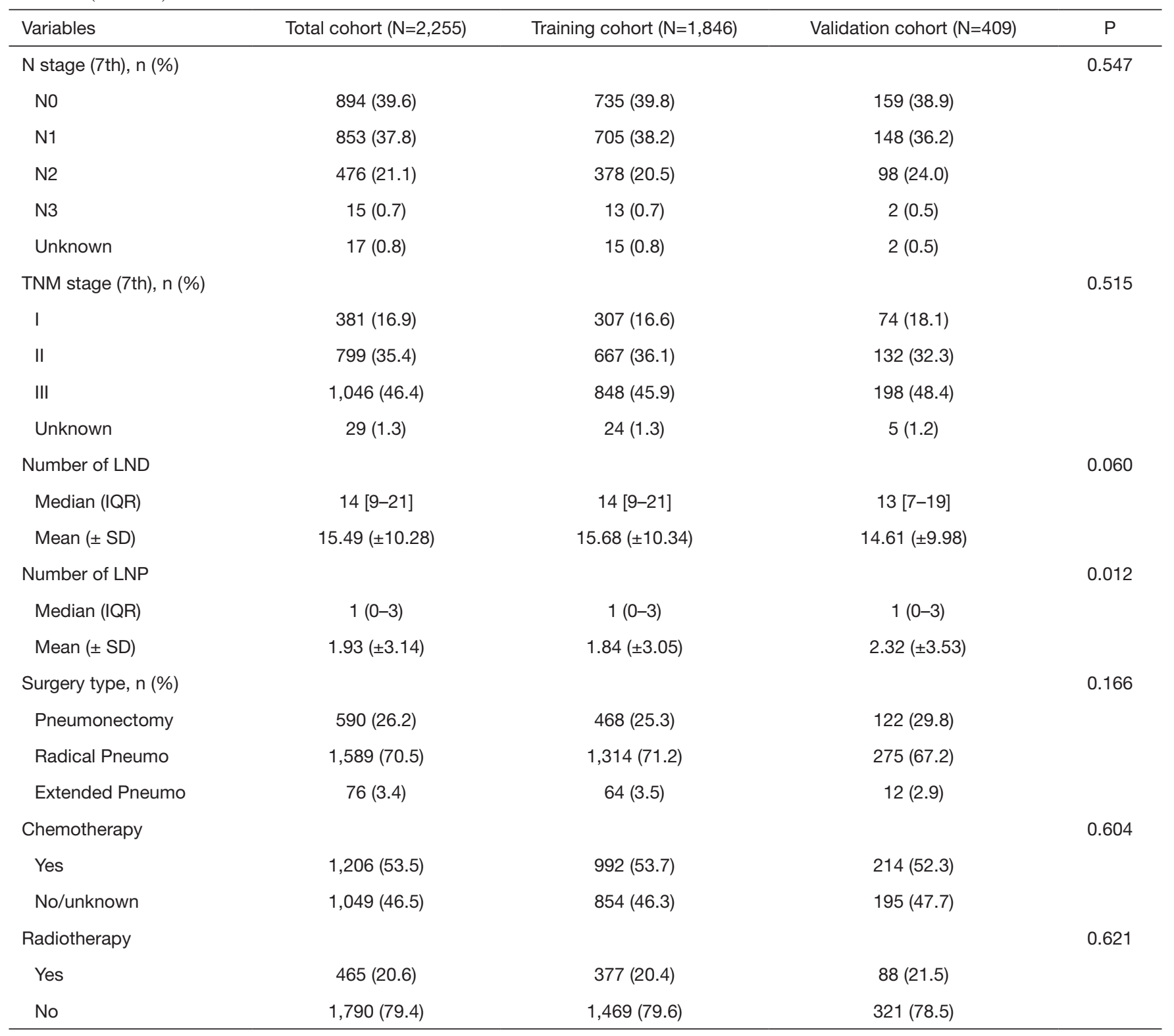

ADC, adenocarcinoma; SQC, squamous cell carcinoma; NEU, neuroendocrine carcinoma; Pneumo, pneumonectomy; LND, lymph nodes dissection; LNP, lymph nodes positive.

radiotherapy were independent factors of long-term survival. The prognostic significant factors were included in the construction of the predictive model for the nomogram (Figure 1A). An online version of the nomogram to assist researchers and clinicians could be accessed at https:// thoracic.shinyapps.io/nomogrampneumonectomy/. Predicted survival probability across time could be easily determined by inputting clinical features and reading output figures and tables generated by the webserver (Figure 1B). The C-index of OS, which indicates discrimination ability, was 0.675 (95\% CI: 0.655-0.694). Similarly, the AUC of the prediction model was $0.733,0.709$, and 0.701 for the 1-, 3-, and 5-year OS, respectively (Figure 2A,B,C). These findings indicated that the nomogram had moderate predictive ability for OS. As shown in Figures $2 D$ and 3, the internal (Figure 2D) and external (Figure 3A,B,C) calibration plots of 
Table 2 OS univariate and multivariate analysis of prognostic factors in the training cohort.

\begin{tabular}{|c|c|c|c|c|}
\hline Variables & \multicolumn{2}{|c|}{ Univariate analysis of OS } & \multicolumn{2}{|c|}{ Multivariate analysis of OS } \\
\hline \multicolumn{5}{|l|}{ Gender } \\
\hline Male vs. female & $1.392(1.204-1.609)$ & $<0.001$ & $1.201(1.021-1.414)$ & 0.027 \\
\hline Age & $1.026(1.019-1.033)$ & $<0.001$ & $1.025(1.017-1.034)$ & $<0.001$ \\
\hline Other vs. Caucasoid & $1.021(0.844-1.236)$ & 0.828 & & \\
\hline \multicolumn{5}{|l|}{ Marriage } \\
\hline Other vs. married & $1.120(0.977-1.286)$ & 0.105 & & \\
\hline Histology & & $<0.001$ & & \\
\hline Other vs. NEC & $2.811(1.819-4.345)$ & $<0.001$ & 2.217 (1.303-3.774) & 0.003 \\
\hline Grading & & $<0.001$ & & \\
\hline II vs. I & $1.606(1.153-2.238)$ & 0.005 & $0.977(0.681-1.402)$ & 0.899 \\
\hline III \& IV vs. I & $2.193(1.587-3.030)$ & $<0.001$ & $1.281(0.896-1.833)$ & 0.175 \\
\hline \multicolumn{5}{|l|}{ Laterality } \\
\hline Right vs. left & $1.379(1.204-1.580)$ & $<0.001$ & $1.472(1.267-1.709)$ & $<0.001$ \\
\hline Tumor size & & $<0.001$ & & \\
\hline$\leq 5$ vs. $\leq 3 \mathrm{~cm}$ & $1.232(1.003-1.513)$ & 0.046 & $1.165(0.931-1.456)$ & 0.182 \\
\hline N3 vs. N0 & $2.809(1.445-5.459)$ & 0.002 & $4.482(2.128-9.438)$ & $<0.001$ \\
\hline Number of LND & $0.998(0.991-1.004)$ & 0.487 & & \\
\hline Number of LNP & $1.033(1.019-1.048)$ & $<0.001$ & $1.016(0.995-1.037)$ & 0.127 \\
\hline Surgery type & & 0.168 & & \\
\hline Radical Pneumo vs. Pneumo & $0.911(0.781-1.063)$ & 0.237 & & \\
\hline Extended Pneumo vs. Pneumo & $1.215(0.843-1.751)$ & 0.297 & & \\
\hline \multicolumn{5}{|l|}{ Chemotherapy } \\
\hline Yes vs. no/unknown & $0.769(0.672-0.880)$ & $<0.001$ & $0.558(0.471-0.660)$ & $<0.001$ \\
\hline \multicolumn{5}{|l|}{ Radiotherapy } \\
\hline Yes vs. no & $1.244(1.061-1.458)$ & 0.007 & $1.428(1.168-1.747)$ & 0.001 \\
\hline
\end{tabular}

OS, overall survival; HR, hazard ratio; Cl, confidence interval; ADC, adenocarcinoma; SQC, squamous cell carcinoma; NEC, neuroendocrine carcinoma; Pneumo, pneumonectomy; LND, lymph nodes dissection; LNP, lymph nodes positive. 
A

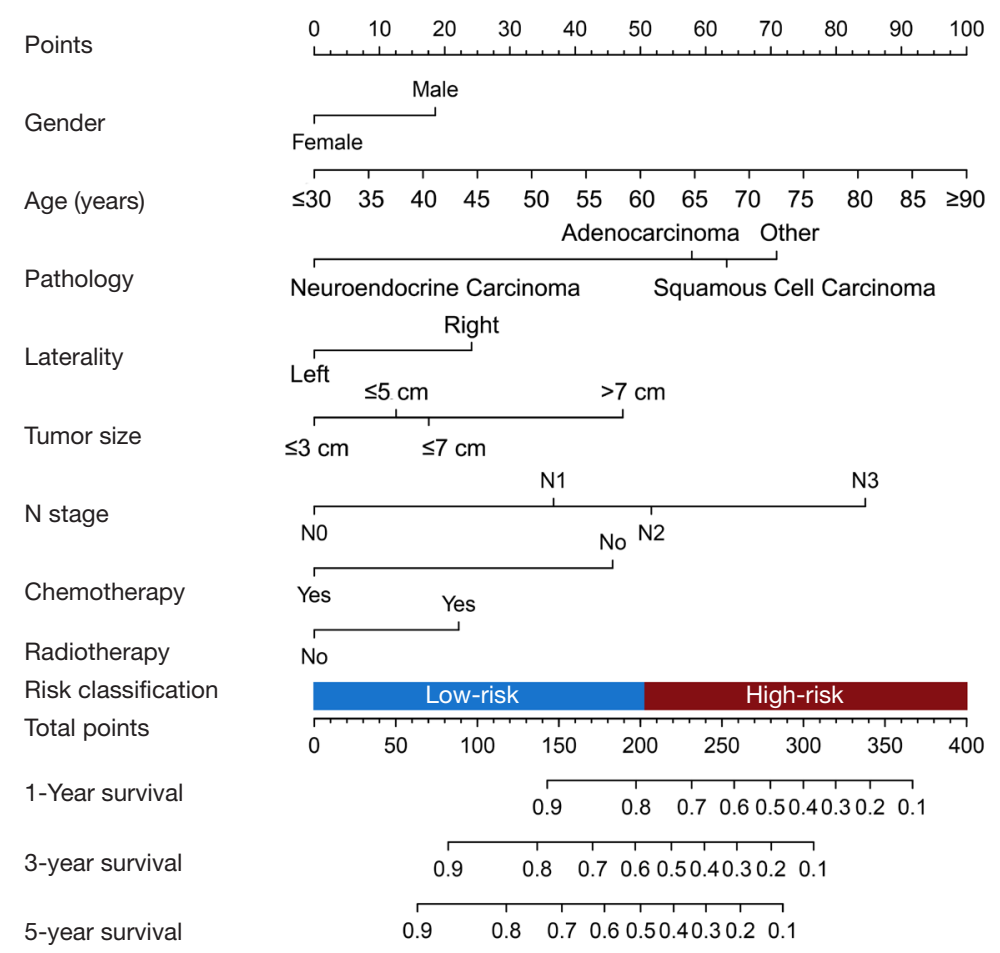

B

Nomogram for patients underwent pneumonectomy

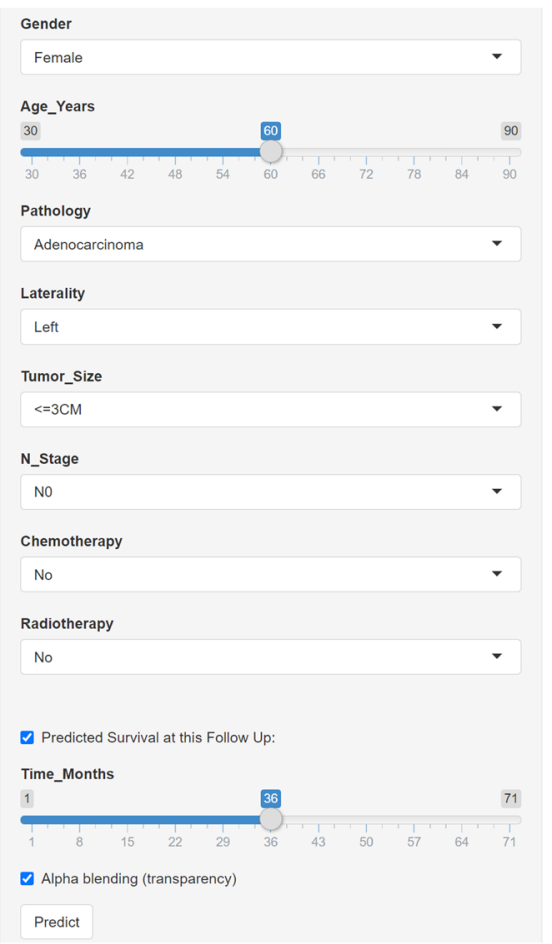

Figure 1 The predictive model developed in this study. Nomogram (A) and online calculation tool (B) predicting the OS for patients undergoing pneumonectomy diagnosed NSCLC. OS, overall survival; NSCLC, non-small cell lung cancer.

the predictive model for the 1-, 3-, and 5-year OS were in good agreement.

\section{Risk classification system}

The novel risk classification system placed patients into the low-risk $(934 / 1,846$; score $\leq 203)$ or high-risk group $(912 / 1,846$; score $>203)$ by the median value of the total points for each patient in the training cohort (Figure 1A). In the total cohort, the medians OS of patients in the low- and high-risk groups were 75.0 and 26.0 months, respectively (Log-rank $\mathrm{P}<0.001$, Figure $4 A$ ). The KaplanMeier curves showed that the OS of the two groups can be significantly differentiated by the risk classification system both in the training and validation cohorts (Logrank $\mathrm{P}<0.001$, Figure $4 B, C$ ). There were also significant survival differences between the low- and high-risk groups in stage I, II, or III lung cancer (Log-rank $\mathrm{P}<0.05$, Figure $5 A, B, C)$. The high-risk patients in stage I and II still had worse survival outcome than those in stage II and III with low-risk (3-year mortality: stage I/high-risk vs. stage II/low-risk: $46.0 \%$ vs. $29.4 \%$, Logrank $\mathrm{P}=0.009$; stage II/high-risk vs. stage III/low-risk: $57.0 \%$ vs. $39.6 \%$, Log-rank $\mathrm{P}<0.001$, Figure $5 D$ ). However, the prognosis difference between patients in stage I/highrisk and stage III/low-risk groups did not show significant statistical differences (5-year mortality: stage I/high-risk vs. stage III/low-risk: $52.9 \%$ vs. $51.8 \%$, Log-rank $\mathrm{P}=0.456$, Figure 5D).

\section{Discussion}

This study focused on the construction of a survival prediction model for patients who had undergone pneumonectomy, which systematically analyzed the clinicopathological factors of patients identified in the SEER database. We obtained multiple independent prognostic variables to build a reliable predictive nomogram, a convenient online tool, and an effective risk classification system. 

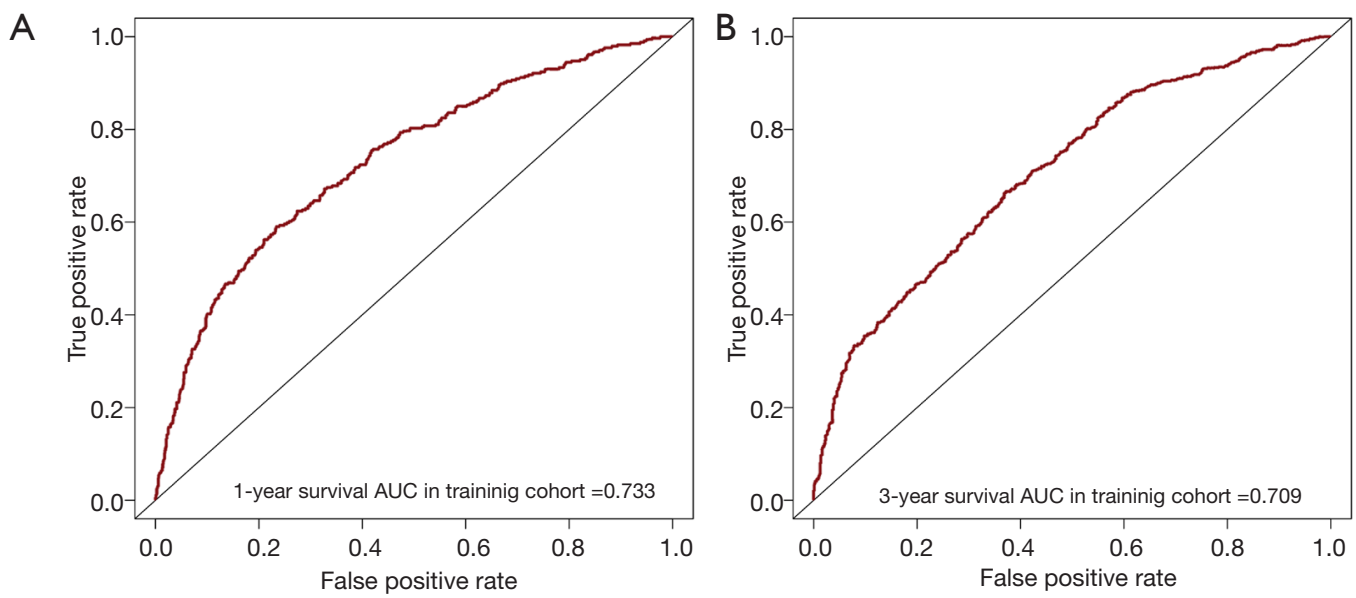

C

\section{D}
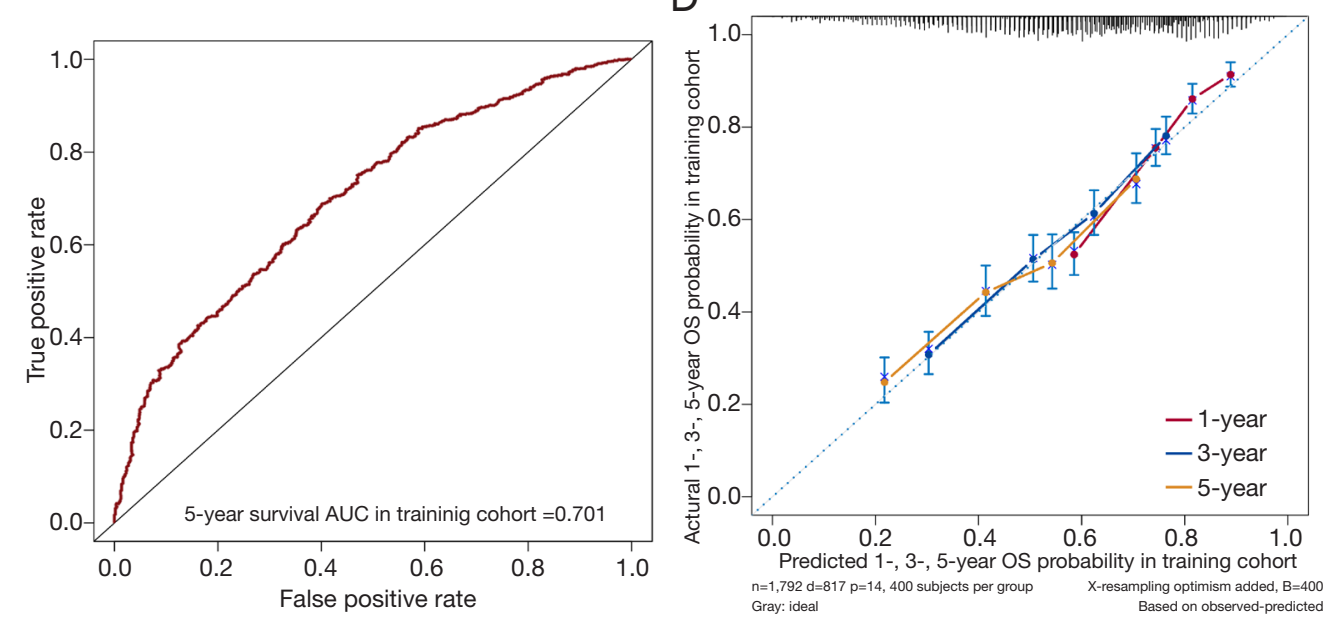

Figure 2 The ROC curve and calibration plots of the predictive model in the training cohort. The area under the ROC curve of 1-, 3-, and 5 -year OS in the training cohort were $0.733,0.709$ and 0.701, respectively (A,B,C); Calibration plots comparing actual and predicted overall survival probabilities at 1-, 3- and 5-year follow-up in the training set (D). Perfect prediction would correspond to a slope of 1 (diagonal 45-degree gray line). ROC, receiver operating characteristic.
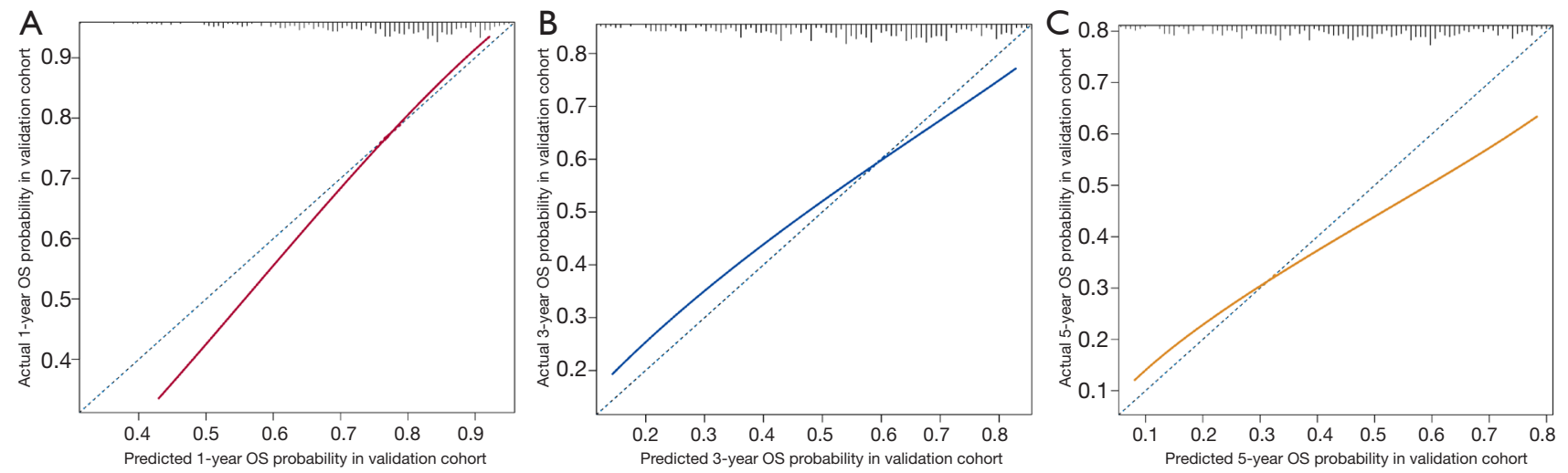

Figure 3 Calibration plots of the predictive model in the validation cohort. Calibration plots comparing actual and predicted overall survival probabilities at 1-, 3- and 5-year (A,B,C) follow-up in the validation set. 

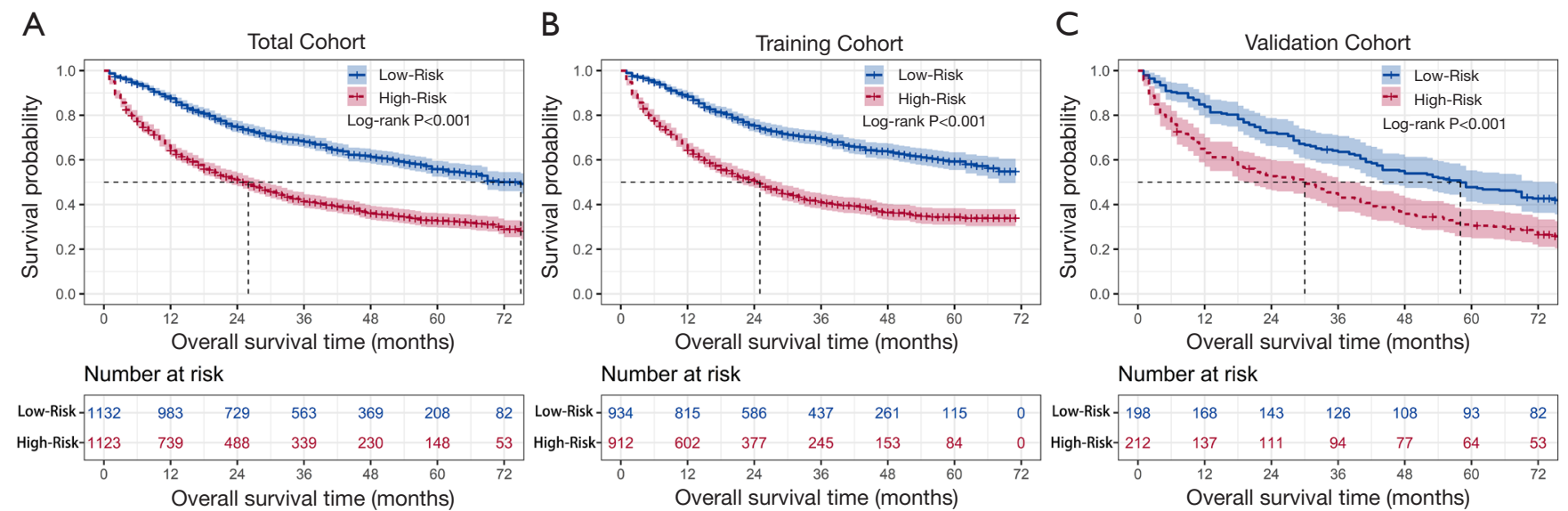

Figure 4 The OS Kaplan-Meier curves of patients in low- and high-risk groups. In the total (A), training (B), and validation (C) cohort, patients in low-risk group had significantly superior OS than the high-risk group (Log-rank $\mathrm{P}<0.001)$. OS, overall survival.

In recent years, there have been improvements in anesthesia, surgical techniques, and perioperative nursing, each contributing to a reduction in mortality associated with pneumonectomy $(15,16)$. Pneumonectomy is a highrisk surgical procedure accounting for about $15 \%$ of lung cancer surgeries (4). During the period of this study, the proportion of pneumonectomy was only $3.9 \%$ among all lung cancer resections and with a reduction tendency during 2011-2015, which was lower than the data from the European Society of Thoracic Surgeons (ESTS) (7.4\%) between 2010 and 2013 (17). Similar studies from English and Danish registries data showed a significant decrease in pneumonectomy proportion over time $(18,19)$. Pneumonectomy will still be used as an inevitable and effective method of treatment for some patients with centrally located or locally advanced lung cancer (20).

Previously, researchers have created the Thoracic Surgery Scoring System (Thoracoscore) using the French Epithor database, to evaluate the prognosis of patients undergoing thoracic surgery $(21,22)$. This system has later been included in the guidelines for the British Thoracic Society (23). However, one study found that Thoracoscore was not reliable in predicting mortality after pneumonectomy because of the under-representation of this high-risk procedure in the database (6\%) (24). Several nomograms for lung cancer have been developed, but few systemically predicted outcomes after pneumonectomy. For instance, Cheng and colleagues constructed a nomogram prediction model for the prognosis of patients undergoing pneumonectomy from a cohort of 100 patients (25). This model incorporated circulating blood biomarkers and tumor characteristics, and demonstrated prognostic superiority over the pTNM staging system. But, the calibration curve of the study did not show good agreement in the discovery and validation cohorts, which is likely due to its small sample size. Compared with the current only nomogram study on patients post pneumonectomy, our research has a bigger sample size, and the nomogram produced good results in both training and validation cohorts. For patients undergoing pneumonectomy in the same cancer stage, the risk classification system can significantly distinguish between the high- and low-risk groups. Considering the small proportion of this highly risk procedure, it was difficult to conduct randomized studies. Therefore, more researches based on the prospective designed database including multicenter was expected to in-depth evaluate the effects of different pathological indicators on short- and long-term prognosis.

In this study, an effective risk classification system was constructed by nomogram prediction model. The prognosis of patients in each stage of lung cancer in high-risk group was significantly worse than that in low-risk group. There was no significant difference in long-term overall mortality between patients in stage I/high-risk group and stage III/ low-risk group. Based on our risk classification system, we could more accurately identify high-risk patients in different stages of lung cancer and provide more rigorous follow-up and care. The authors also provided an online version of the dynamic nomogram which was easier-to-use for physicians to predict the long-term survival of patients with different clinicopathological features.

Pneumonectomy laterality has an impact on the long- 
A
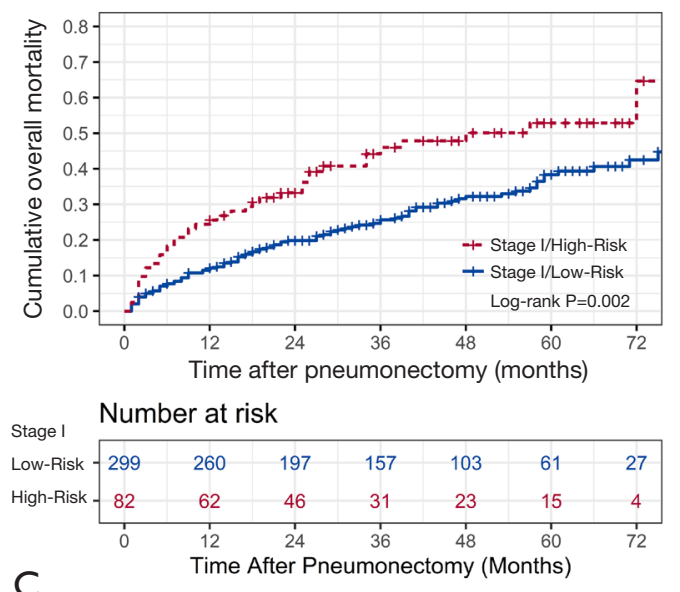

C

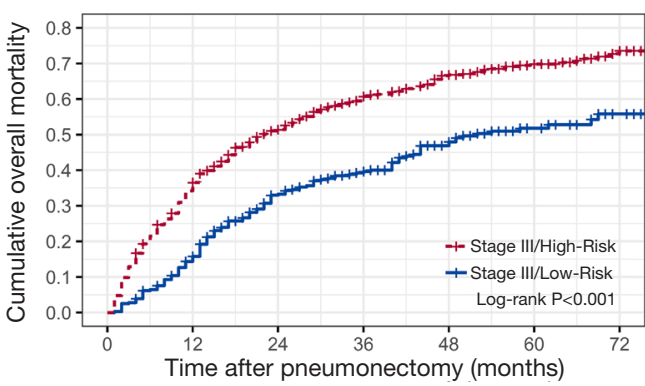

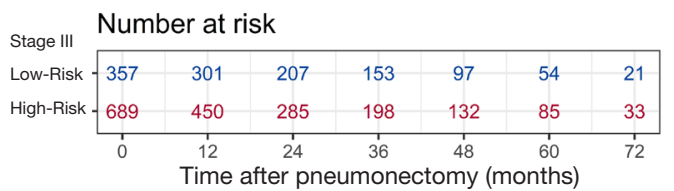

$\mathrm{B}$

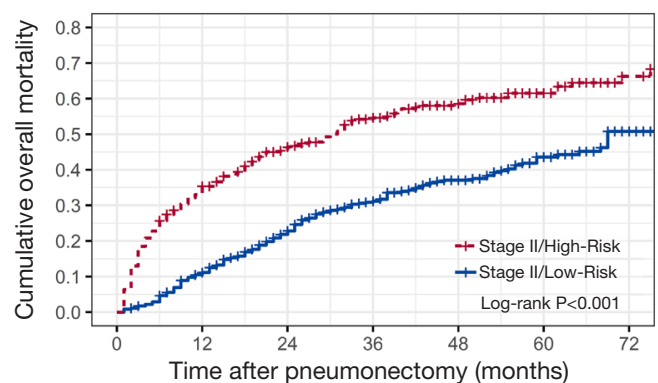

Number at risk

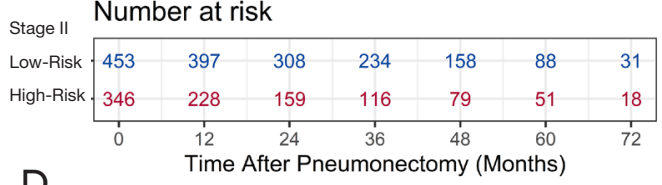

D
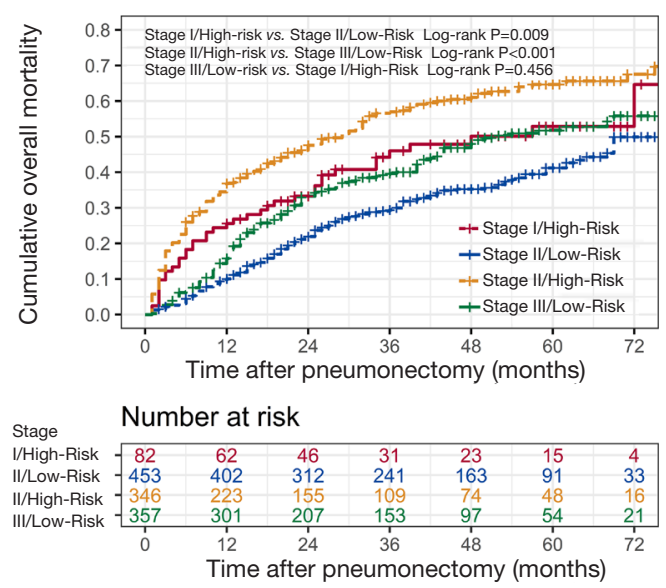

Figure 5 The cumulative overall mortality of patients in different stages. Patients with low-risk had significantly better survival than patients with high-risk in TNM stage I (A), II (B), and III (C) (Log-rank $\mathrm{P}<0.05)$. The patients in stage I and II with high-risk had significantly worse survival than stage II and III with low-risk (Stage I/high-risk vs. Stage II/low-risk, Log-rank P=0.009; Stage II/high-risk vs. Stage III/lowrisk, Log-rank $\mathrm{P}<0.001)$, but the patients in stage $\mathrm{I} /$ high-risk and stage III/low-risk groups had similar cumulative overall mortality without significant statistical difference (Log-rank $\mathrm{P}=0.456)(\mathrm{D})$.

term survival of patients. In this study, we found that patients with a right-side pneumonectomy presented worse prognosis than those with a left-side pneumonectomy. This observation may be related to several factors. First, $55 \%$ of ventilation is performed by the right lung; right-side pneumonectomy will thus proportionately affect the overall lung function (1). Secondly, mediastinal displacement with the asymmetric position of the heart to the left also results in relief of the left side after pneumonectomy. The right side lacks a corresponding resistance to mediastinal displacement, which may have a negative impact on hemodynamic stability and pose a possible life-threatening risk (26). In addition, some researchers also reported that the proportion of serious postoperative complications is higher on the right side (acute respiratory distress syndrome and bronchopleural fistula), which leads to higher morbidity and mortality. This is due to a lack of retraction on the right stump as opposed to the left, which makes the right stump more prone to inflammation and breakdown (27).

In this study, we found that the use of chemotherapy improved OS of patients while the radiotherapy showed a negative effect. Possible explanation may be related to the fact that chemotherapy serves as a more systemic treatment, while radiotherapy is a local treatment. In addition, radiotherapy can increase the burden on cardiopulmonary function, leading to serious complications and reduced longterm survival. Researchers also found that chemotherapy improves the prognosis of patients with pneumonectomy (28). 
Kim et al. demonstrated that forced expiratory volume in 1 second (FEV1) at the beginning of postoperative radiotherapy - that is closely related to the extent of resection-was the only significant prognostic factor for OS (29). Given that patients undergoing pneumonectomy usually have reduced lung function, Karnofsky performance status scores and cardiopulmonary tolerance should be carefully evaluated and optimized when considering radiotherapy or chemotherapy.

However, the current study has several limitations. First, this study was a retrospective study with common defects. Second, only the variables available in the SEER database could be analyzed with no access to more detailed information such as the Charlson comorbidity index, pulmonary function evaluation, postoperative complications, the chemotherapy regimen, and the surgery approaches (e.g., open or videoassisted thorascopic surgery). The missing information also brought effects on the accuracy of the nomogram. Third, due to the lack of tumor specific site and invading information, we are unable to fully utilize the 8th staging $\mathrm{p} T$ criteria to evaluate the primary tumor in the prediction model.

Pneumonectomy accounted for about 3.9\% of lung cancer operations in the same period. The nomogram was developed from a number of independent prognostic clinicopathological variables and demonstrated its good utility in C-index and AUC results. The predictive model also had a satisfactory fit to the calibration curves with both internal and external verification. The nomogram and online calculator have essential practical significance in clinical settings, which can help physicians to evaluate the long-term survival of patients and optimize the personalized treatments for lung cancer.

\section{Acknowledgments}

Funding: None.

\section{Footnote}

Reporting Checklist: The authors have completed the TRIPOD reporting checklist. Available at http://dx.doi. org/10.21037/jtd-20-3203

Peer Review File: Available at http://dx.doi.org/10.21037/jtd20-3203

Conflicts of Interest: All authors have completed the ICMJE uniform disclosure form (available at http://dx.doi. org/10.21037/jtd-20-3203). MI serves as an unpaid editorial board member of Journal of Thoracic Disease from Aug 2019 to Jul 2021. DGR serves as an unpaid editorial board member of Fournal of Thoracic Disease from Jan 2020 to Dec 2021. The other authors have no conflicts of interest to declare.

Ethical Statement: The authors declare that they have no conflicts of interest. The authors are accountable for all aspects of the work in ensuring that questions related to the accuracy or integrity of any part of the work are appropriately investigated and resolved. The study was conducted in accordance with the Declaration of Helsinki (as revised in 2013).

Open Access Statement: This is an Open Access article distributed in accordance with the Creative Commons Attribution-NonCommercial-NoDerivs 4.0 International License (CC BY-NC-ND 4.0), which permits the noncommercial replication and distribution of the article with the strict proviso that no changes or edits are made and the original work is properly cited (including links to both the formal publication through the relevant DOI and the license). See: https://creativecommons.org/licenses/by-nc-nd/4.0/.

\section{References}

1. Fuentes PA. Pneumonectomy: historical perspective and prospective insight. Eur J Cardiothorac Surg 2003;23:439-45.

2. Chen J, Soultanis KM, Sun F, et al. Outcomes of sleeve lobectomy versus pneumonectomy: A propensity scorematched study. J Thorac Cardiovasc Surg 2020. [Epub ahead of print]. doi: 10.1016/j.jtcvs.2020.08.027.

3. Licker M, Spiliopoulos A, Frey JG, et al. Risk factors for early mortality and major complications following pneumonectomy for non-small cell carcinoma of the lung. Chest 2002;121:1890-7.

4. Petrella F, Spaggiari L. Therapeutic options following pneumonectomy in non-small cell lung cancer. Expert Rev Respir Med 2016;10:919-25.

5. Kearney DJ, Lee TH, Reilly JJ, et al. Assessment of operative risk in patients undergoing lung resection. Importance of predicted pulmonary function. Chest 1994;105:753-9.

6. Gebitekin C, Martin PG, Satur CM, et al. Results of pneumonectomy for cancer in patients with limited ventilatory function. Eur J Cardiothorac Surg 1995;9:347-51. 
7. Lanuti M. Commentary: Comparing sleeve lobectomy with pneumonectomy for central lung cancer: A randomized study will never be conducted. J Thorac Cardiovasc Surg 2020. [Epub ahead of print]. doi: 10.1016/ j.jtcvs.2020.10.014.

8. Janet-Vendroux A, Loi M, Bobbio A, et al. Which is the Role of Pneumonectomy in the Era of ParenchymalSparing Procedures? Early/Long-Term Survival and Functional Results of a Single-Center Experience. Lung 2015;193:965-73.

9. Powell ES, Pearce AC, Cook D, et al. UK pneumonectomy outcome study (UKPOS): a prospective observational study of pneumonectomy outcome. J Cardiothorac Surg 2009; 4:41.

10. Balachandran VP, Gonen M, Smith JJ, et al. Nomograms in oncology: more than meets the eye. Lancet Oncol 2015;16:e173-80.

11. Iasonos A, Schrag D, Raj GV, et al. How to build and interpret a nomogram for cancer prognosis. J Clin Oncol 2008;26:1364-70.

12. Wang Y, Li J, Xia Y, et al. Prognostic nomogram for intrahepatic cholangiocarcinoma after partial hepatectomy. J Clin Oncol 2013;31:1188-95.

13. Travis WD, Brambilla E, Nicholson AG, et al. The 2015 World Health Organization Classification of Lung Tumors: Impact of Genetic, Clinical and Radiologic Advances Since the 2004 Classification. J Thorac Oncol 2015;10:1243-60.

14. Collins GS, Reitsma JB, Altman DG, et al. Transparent reporting of a multivariable prediction model for individual prognosis or diagnosis (TRIPOD): the TRIPOD Statement. BMC Med 2015;13:1.

15. Duque JL, Ramos G, Castrodeza J, et al. Early complications in surgical treatment of lung cancer: a prospective, multicenter study. Grupo Cooperativo de Carcinoma Broncogenico de la Sociedad Espanola de Neumologia y Cirugia Toracica. Ann Thorac Surg 1997;63:944-50.

16. Ichiki Y, Nagashima A, Chikaishi Y, et al. Pneumonectomy for non-small cell lung cancer. Surg Today 2012;42:830-4.

17. Seder CW, Salati M, Kozower BD, et al. Variation in Pulmonary Resection Practices Between The Society of Thoracic Surgeons and the European Society of Thoracic Surgeons General Thoracic Surgery Databases. Ann Thorac Surg 2016;101:2077-84.

18. Jakobsen E, Palshof T, Osterlind K, et al. Data from a national lung cancer registry contributes to improve outcome and quality of surgery: Danish results. Eur J Cardiothorac Surg 2009;35:348-52; discussion 352.
19. Riaz SP, Linklater KM, Page R, et al. Recent trends in resection rates among non-small cell lung cancer patients in England. Thorax 2012;67:811-4.

20. Pricopi C, Mordant P, Rivera C, et al. Postoperative morbidity and mortality after pneumonectomy: a 30year experience of 2064 consecutive patients. Interact Cardiovasc Thorac Surg 2015;20:316-21.

21. Bernard A, Rivera C, Pages PB, et al. Risk model of inhospital mortality after pulmonary resection for cancer: a national database of the French Society of Thoracic and Cardiovascular Surgery (Epithor). J Thorac Cardiovasc Surg 2011;141:449-58.

22. Falcoz PE, Conti M, Brouchet L, et al. The Thoracic Surgery Scoring System (Thoracoscore): risk model for in-hospital death in 15,183 patients requiring thoracic surgery. J Thorac Cardiovasc Surg 2007;133:325-32.

23. Lim E, Baldwin D, Beckles M, et al. Guidelines on the radical management of patients with lung cancer. Thorax 2010;65 Suppl 3:iii1-27.

24. Qadri SS, Jarvis M, Ariyaratnam P, et al. Could Thoracoscore predict postoperative mortality in patients undergoing pneumonectomy? Eur J Cardiothorac Surg 2014;45:864-9.

25. Cheng B, Wang C, Zou B, et al. A nomogram to predict outcomes of lung cancer patients after pneumonectomy based on 47 indicators. Cancer Med 2020;9:1430-40.

26. Bazwinsky-Wutschke I, Paulsen F, Stovesandt D, et al. Anatomical changes after pneumonectomy. Ann Anat 2011;193:168-72.

27. Sugarbaker DJ, Haywood-Watson RJ, Wald O. Pneumonectomy for Non-Small Cell Lung Cancer. Surg Oncol Clin N Am 2016;25:533-51.

28. Thibout Y, Guibert B, Bossard N, et al. Is pneumonectomy after induction chemotherapy for non-small cell lung cancer a reasonable procedure? A multicenter retrospective study of 228 cases. J Thorac Oncol 2009;4:1496-503.

29. Kim H, Lussier YA, Noh OK, et al. Prognostic implication of pulmonary function at the beginning of postoperative radiotherapy in non-small cell lung cancer. Radiother Oncol 2014;113:374-8.

Cite this article as: Wang ZM, Swierzy M, Balke D, Nachira D, González-Rivas D, Badakhshi H, Ismail M. Dynamic nomogram for long-term survival in patients with non-small cell lung cancer after pneumonectomy. J Thorac Dis 2021;13(4):22762287. doi: $10.21037 /$ jtd-20-3203 